\title{
Societal Burden and Persisting Unmet Needs of Parkinson's Disease
}

\author{
K Ray Chaudhuri1,2 and Nataliya Titova ${ }^{3}$ \\ 1. National Parkinson Foundation Centre of Excellence, King's College Hospital, Denmark Hill, London, UK; 2. King's College London, \\ Department of Neurosciences, Institute of Psychiatry, Psychology \& Neuroscience, London, UK; 3. Department of Neurology, Neurosurgery \\ and Medical Genetics, Pirogov Russian National Research Medical University, Moscow, Russian Federation
}

$\mathrm{P}$ arkinson's disease (PD) imposes a serious burden on patients, carers, families, healthcare providers and health authorities globally. PD affects $0.3 \%$ of people in the developed world and its prevalence is increasing with an estimated 2.1 million people with PD worldwide in 1990 rising to 6.1 million in 2016. This increase has been driven by growing elderly populations and other, as yet, unknown factors. The cost of PD has been estimated at $\$ 14.4$ billion/year in the USA and that is predicted to double by 2040. PD continues to have manifold unmet needs in terms of understanding pathophysiology, early detection, reliable biochemical and/or genetic biomarkers, diagnosis, improved PD assessment scales, predicting trajectory, and effective treatments for both motor and non-motor symptoms. Levodopa continues to be the most effective drug treatment for PD; in recent decades, many promising improved drug therapies have failed to show efficacy in clinical trials. The treatments available mainly address motor symptoms but not the non-motor symptoms which often start earlier and are overall more detrimental to patient well-being and quality of life. Holistic approaches with multiple modes of action are needed in the development of new treatments rather than using single target approaches such as drugs that only affect the dopaminergic system. Despite this apparently bleak overall picture, there are grounds for optimism as knowledge of PD pathophysiology is increasing, potential new therapeutic targets have been identified and novel treatments are in development. Earlier diagnosis, treating non-motor symptoms as they appear and the ongoing development of drugs to slow or alter disease progression have the potential to improve the outlook for people with PD at present or in the near future.

\section{Keywords}

Parkinson's disease, patient burden, economic burden, societal burden, unmet needs

Disclosures: K Ray Chaudhuri has participated on scientific advisory boards and/or received honoraria for scientific advice from Abbott, Boehringer-Ingelheim, GSK, Serono, Teva, UCB and Britannia Pharmaceuticals. Nataliya Titova has received honoraria for lectures in educational symposia from Teva and UCB. Review Process: Double-blind peer review. Acknowledgement: Medical writing support, including preparation of the drafts under the guidance of the author, was provided by James Gilbart, Freelance Writer for Touch Medical Media, and was supported by Touch Medical Media.

Compliance with Ethics: This study involves a review of the literature and did not involve any studies with human or animal subjects performed by any of the authors.

Authorship: The named authors meet the criteria of the International Committee of Medical Journal Editors for authorship for this manuscript, take responsibility for the integrity of the work as a whole, and have given final approval for the version to be published.

Received: 25 May 2019

Accepted: 12 June 2019

Citation: European Neurological Review. 2019;14(1):28-35

Corresponding Author: K Ray Chaudhuri,

Department of Basic and Clinical Neuroscience, The

Maurice Wohl Clinical Neuroscience Institute, King's

College London, Cutcombe Road, London, SE5 9RT, UK.

E: ray.chaudhuri@kcl.ac.uk Twitter: KRayChaudhuri1

Support: No funding was received for

the publication of this article.
The burdens of Parkinson's disease (PD) are undeniably serious and increasing while various unmet needs remain, especially the absence of disease-modifying therapies and the difficulty of finding new treatments that are effective in patients. ${ }^{1-7}$ Although this seems a discouraging situation, considerable advances in understanding the pathophysiology of PD and the identification of new targets for treatments suggest that the situation is improving. ${ }^{3,8-10}$ In addition, better use of available protocols and technologies have the potential to provide earlier detection of PD and initiate palliative treatments sooner particularly for the non-motor symptoms which have as much, or greater, impact on people with PD than motor symptoms which, until now, have been the primary focus of physicians and the treatments they prescribe. ${ }^{11,12}$ This review considers the current and future burden of PD and the unmet needs in PD, and ongoing progress made in alleviating them.

\section{The societal burden of Parkinson's disease - associated with putative aetiologies and pathophysiology}

PD exerts a heavy societal burden worldwide and the rising prevalence with an increasingly ageing population has rendered this condition to be the fastest growing neurodegenerative condition and one of the most expensive disorders in this sense., 5,13,14 The toll on patients, caregivers and treatment providers has been increasing in recent decades while the continuing failures of neuroprotection trials, and consequent lack of disease-modifying treatments and insufficiently effective therapies for its numerous non-motor symptoms, continue as key unmet needs. ${ }^{4,15,16}$ Levodopa remains the most effective drug for PD in spite of millions being spent in new drug development. $3,4,17-19$ PD is the second most common neurodegenerative disease (after Alzheimer's disease), affecting $0.3 \%$ of people in the developed world rising to $3 \%$ of those aged $>65$ years. ${ }^{20}$ The Global Burden of Disease (GBD) Collaboration systematic analysis estimated that there were 211,296 deaths ascribed to a diagnosis of PD in 2016; although the condition itself is not fatal. It also estimated that there were 6.1 million people with PD worldwide in 2016, compared with 2.1 million in 1990.1 There were greater proportions with PD in regions with a high income (e.g. Canada, US, Western Europe, 90-180/100,000) compared with low income (e.g. sub-Saharan Africa, 30-70/100,000) (Figure 1).? The GBD analysis also estimated PD prevalence according to socio-demographic index (SDI, a composite measure of income per capita, education and fertility) and reported that there were 2.1 million people with PD in high SDI regions, 1.8 million in middle SDI regions and 113,00 in low SDI regions. PD was estimated to cause 3.2 million disability-adjusted life years and this impact was greatest in high SDI countries (Figure 2). PD also has unequal gender and racial impact being 
Figure 1: Age-standardised prevalence of Parkinson's disease per 100,000 population for both sexes in 2016

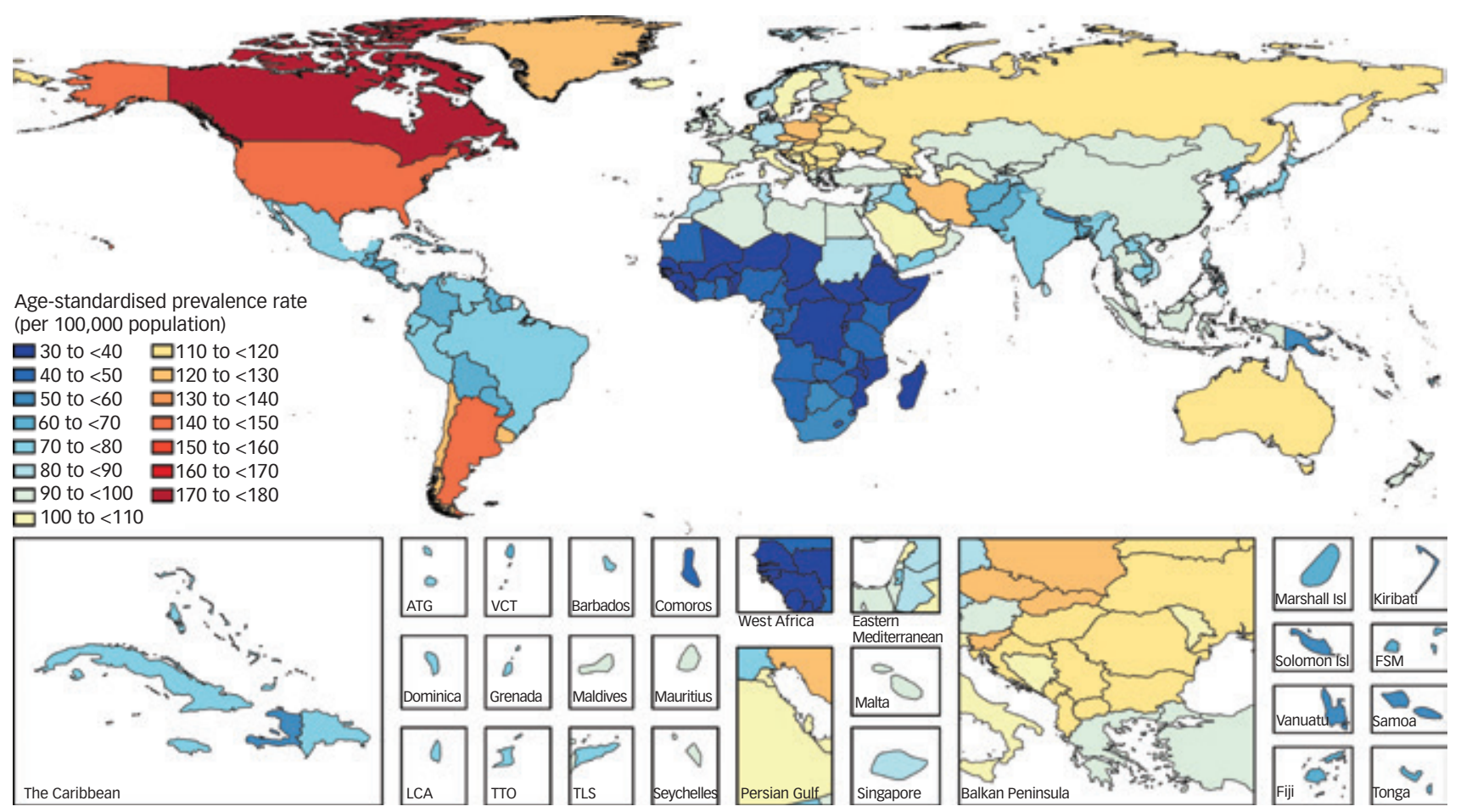

ATG = Antigua and Barbuda; FSM = Federated States of Micronesia; ISI = ISlands; $L C A=$ Saint Lucia; TLS = Timor-Leste; TTO = Trinidad and Tobago; VCT = Saint Vincent and the Grenadines. Reproduced with permission from the Global Burden of Disease Parkinson's Disease Collaborators 2016.1

Figure 2: Age-standardised DALY rates for Parkinson's disease in 21 regions by socio-demographic index for years 1990-2016

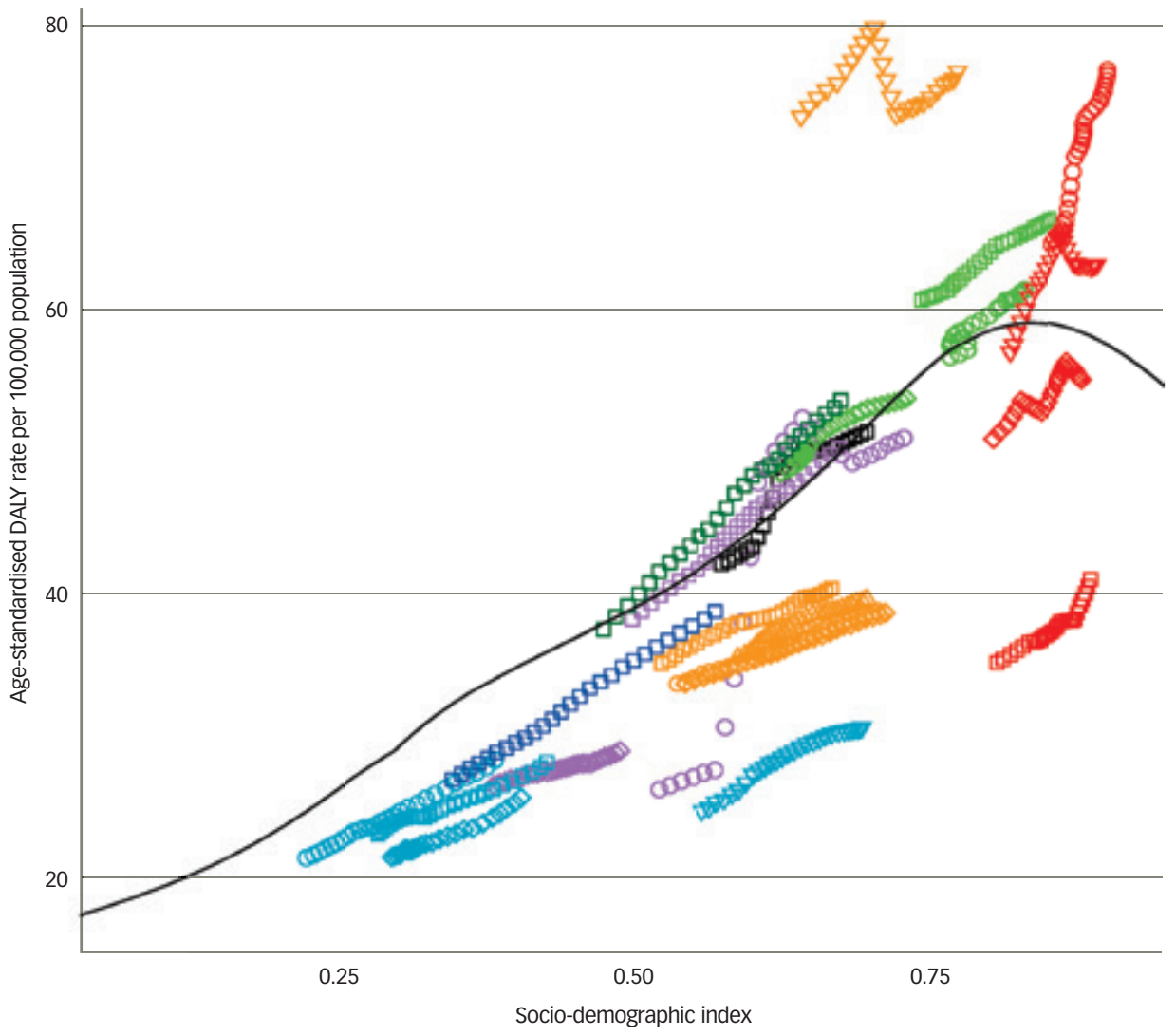

ㅁ Global

․ High-income Asia Pacific

O High-income North America

Western Europe

$\nabla$ Australasia

$\square$ Andean Latin America

Tropical Latin America

$\checkmark$ Central Latin America

$\nabla$ Southern Latin America

$\triangle$ Caribbean

$\square$ Central Europe

- Eastern Europe

$\checkmark$ Central Asia

ㅁ North Africa and Middle East

口 South Asia

$\square$ Southeast Asia

- East Asia

$\checkmark$ Oceania

$\square$ Western sub-Saharan Africa

- Eastern sub-Saharan Africa

$\checkmark$ Central sub-Saharan Africa

$\nabla$ Southern sub-Saharan Africa 
more common in men, Hispanics and white Hispanics. ${ }^{21}$ In 2016, the male:female ratio for PD was estimated to be 1.40 , which was largely unchanged from 1990. ${ }^{1}$ Animal models and clinical investigation findings suggest that this sex disparity could result from differences in the nigrostriatal dopaminergic pathway that may be protective in the female brain. ${ }^{22}$ Better understanding and exploitation of this effect could lead to new therapeutic approaches.

The aetiology of PD remains unclear but is considered to be multifactorial and heterogeneous. The rising population of elderly people worldwide is an important factor contributing to the increased prevalence of PD and its burden because age above $65-70$ years is a well-established risk factor. ${ }^{23}$ However, the GBD analysis showed that over the past 25 years, the age-standardised prevalence rate increased by $21.7 \%$, whereas the crude prevalence rate increased by $74.3 \%$ suggesting additional influences must also be operational. ${ }^{1}$ Various historical, lifestyle and demographic parameters have been investigated as PD risk factors; some have shown some significant associations. These include: anxiety or depression, use of beta-blockers, head injury, lack of physical activity, and high serum uric acid (also blood levels of cholesterol, vitamin D3, insulin-like growth factor 1 [IGF-1], glycated haemoglobin [Hb1AC], C-reactive protein [CRP] levels); although it is not yet clear whether these factors are cause or effect. ${ }^{24}$ Baseline factors including male sex, orthostatic blood pressure drop, diagnosis of coronary artery disease, arterial hypertension, elevated serum uric acid, and cerebrospinal fluid neurofilament light chain have been associated with disease progression after 4 years. ${ }^{25}$ Of interest, smoking has been reported to result in a $74 \%$ reduction in PD risk and some components of tobacco are believed to have a protective effect. ${ }^{26}$ It is possible that the declines in smoking rates starting from the 1940s-50s onwards have actually contributed to increase PD prevalence many decades later. ${ }^{23,24}$ This link, however, may be spurious and has been insufficiently investigated to draw reliable conclusions.

Various studies and meta-analyses have shown statistically significant associations between PD risk and environmental factors such as chronic exposure to agricultural chemicals (insecticides, organochlorines, organophosphates), solvents and metals (especially lead). ${ }^{27-29}$ Thus, workers in farming, metallurgy and textiles are believed to have increased risk; although more epidemiological studies are necessary to substantiate these risks and determine whether they actually contribute to increasing prevalence. An emerging proposed aetiology in PD is the gut-brain hypothesis. ${ }^{30-32}$ This putative mechanism is gaining interest and suggests that microbial disturbances in the gut and consequent T cell-driven inflammation, lead to misfolding of $\alpha$-synuclein, which is a key component of Lewy bodies. PD has been positively correlated with inflammatory bowel diseases which further supports this hypothesis. ${ }^{33}$ It is therefore possible that inflammation mediated by $T$ cells, which produces dopaminergic neurodegeneration in $\mathrm{PD}$, is initiated by a disturbed gut mucosa. This aetiology is intriguing but is not supported by post-mortem evidence ${ }^{34}$ and requires much further investigation. If substantiated, it could lead to development of novel approaches to PD treatment and prevention

Genetics plays a small but important part in PD aetiology. Epidemiological studies during the past 15 years showed there is increased risk of PD among siblings of patients and reported heritability in 10-30\% of PD cases, which is higher than was previously thought.35,36 Mutations associated with autosomal dominant PD include those of the $\alpha$-synuclein (associated with Lewy bodies), leucine-rich repeat kinase 2 (LRRK2, prominent in Ashkenazy Jewish, Arab-Berber and Basque populations), glucocerebrosidase (the commonest mutation and prominent in
Ashkenazy Jewish populations) and vascular sorting protein. ${ }^{36,37} \mathrm{~A}$ different set of mutations are associated with autosomal recessive juvenile PD including mutations in parkin protein (ubiquitin E3 ligase), PTEN-induced ligase 1 (serine-threonine kinase) and Daisuke-Junko-1. ${ }^{36}$ These genetic findings have the potential to increase understanding of PD aetiology and could provide potential new approaches for disease-modifying therapies; but the efficacy of drugs designed to exploit them remains to be demonstrated. ${ }^{9,10}$ In addition, research in pathogenic mutation carriers (LRRK2, glucocerebrosidase) needs to be developed. ${ }^{38}$ Genetic mutations also show an ethnic clustering, for instance, the power to detect the LRRK2 G2019S mutation is 35\% in North African subjects as compared with $1 \%$ in British subjects. ${ }^{39}$

In PD, increasing evidence suggests that the misfolding of $\alpha$-synuclein involves the downregulation or dysfunction of mechanisms of proteostasis, particularly the molecular chaperones. ${ }^{40}$ These are a large group of different heat-shock proteins/factors (HSP/HSF) that control the folding of nascent polypeptide chains, refold misfolded proteins, or mediate the destruction of other misfolded proteins. chaperones provide a valuable target in the development of disease-modifying drugs for PD. Several chaperone-targeted therapies such as HSF-1 modulators, HSP-90 inhibitors, chemical and pharmacological chaperones, and gene therapy to modulate mutated chaperones show promise in the treatment of PD. ${ }^{40}$ In particular, repurposing of ambroxol, a secretolytic agent used for the treatment of bronchopulmonary disorders has shown potential as a possible disease-modifying therapy for PD by increasing glucosylceramidase activity and acts to correct the function of mutated glucocerebrosidase which has a role in $\alpha$-synuclein aggregation. ${ }^{41-43}$ In patients with PD with a relevant glucocerebrosidase mutation, this treatment has the exciting potential of modifying the risk of PD development in asymptomatic individuals and treating existing PD.

More recently, the pathophysiological and clinical heterogeneity of motor and non-motor aspects in different patients have prompted calls for PD to be considered as a syndrome rather than a disease. ${ }^{44}$ This complexity increases the challenge of understanding the disease processes and developing treatments. In PD, there is generally lower awareness and attention paid to the non-motor symptoms which are almost universal and substantially add to the disease burden. ${ }^{11,45,46}$ These commonly include symptoms of autonomic dysfunction including seborrhoea, excessive salivation, orthostatic hypotension, urogenital dysfunction/urinary incontinence and gastrointestinal disturbance (e.g. constipation). Sensory dysfunction (e.g. loss of sense of smell) is also characteristic of $\mathrm{PD}$, as well as pain, which is frequently severe and greatly increases the burden. In addition, a range of neuropsychiatric symptoms also occur in the majority of patients including fatigue, insomnia, daytime sleepiness, behavioural disorders, mood changes, anxiety, apathy, anhedonia, depression, cognitive dysfunction, and hallucinations. ${ }^{45-47}$ Psychosis appears to be a marker for PD progression and is often induced by dopamine replacement therapy, while dementia is common but is not an inevitable result of progression over 15-20 years. ${ }^{45,48}$ Evidence-based treatments for nonmotor symptoms are substantially lacking and the recent Movement Disorders Society non-motor symptoms task force for evidencebased treatment shows lack of level 1 evidence for treatment of the majority of the key non-motor symptoms. ${ }^{49}$ In addition, dopaminergic replacement therapies can themselves promote the occurrence of non-motor symptoms and behavioural issues such as impulse control disorders and dopamine dysregulation syndrome and there are issues with drug interactions in many individuals receiving polypharmacy. ${ }^{50}$ 
Figure 3: Time courses of the onset of the motor and non-motor features of Parkinson's disease

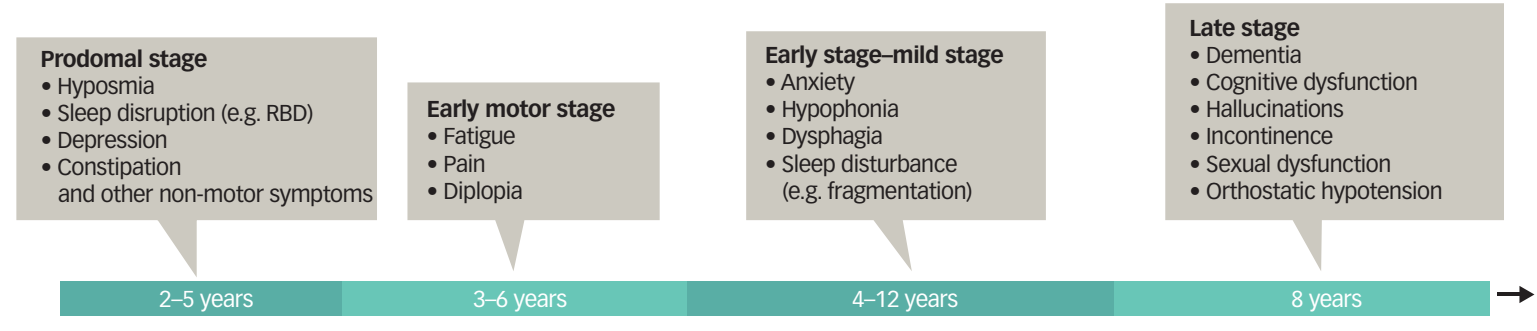

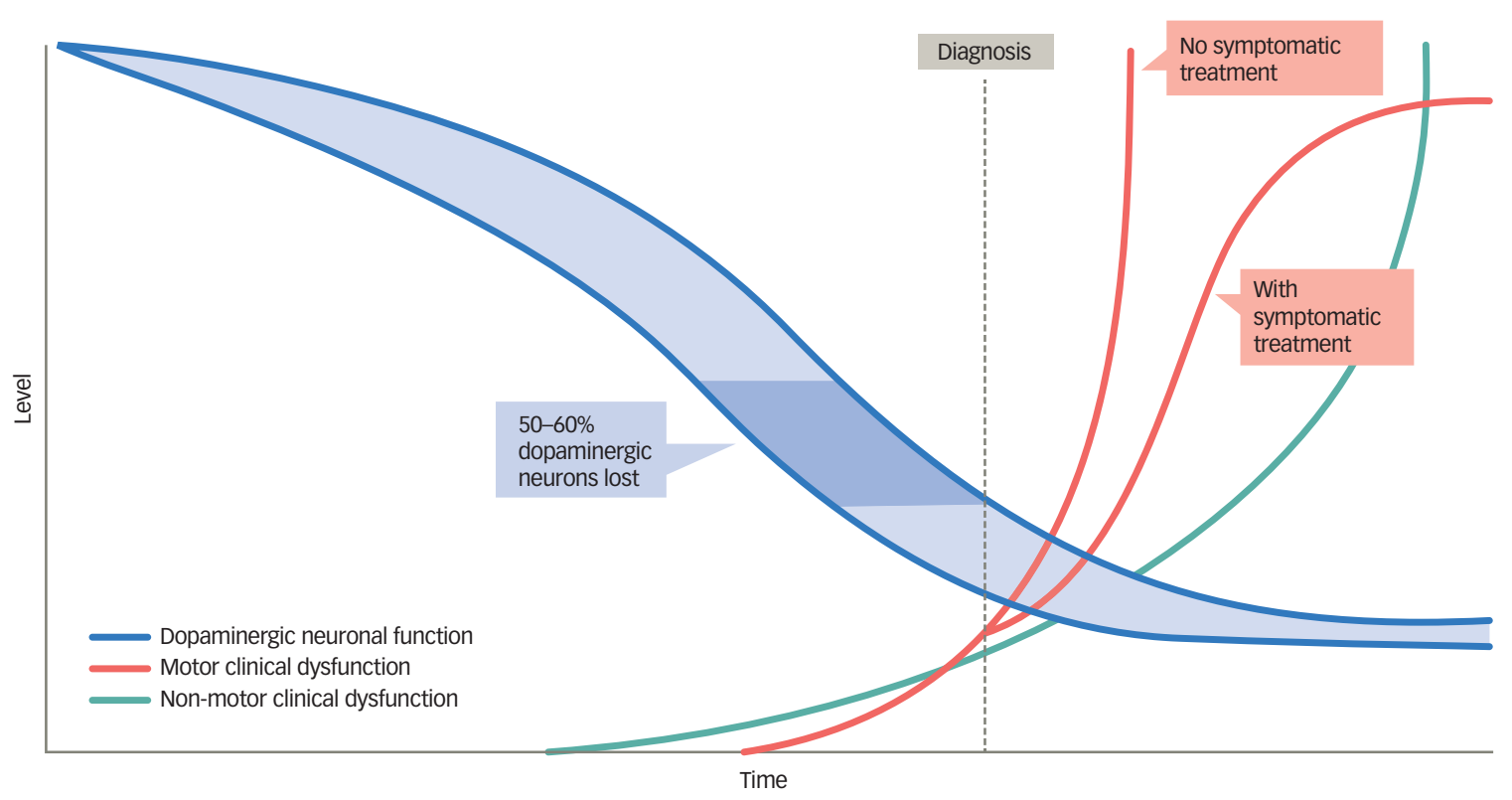

$R B D=$ rapid eye movement sleep behaviour disorder. Reproduced with permission from schapira et al. 2017.62

Non-motor symptoms have a serious impact on the patient with PD and often manifest early when dopaminergic neurones have begun to decline but before motor symptoms have appeared (Figure 3). These symptoms also increase caregiver burden and reduce their quality of life to a significantly greater extent than the motor symptoms. ${ }^{51}$ In one survey, the caregivers of patients with PD $(n=109)$ were found to spend an average 22 hours per week providing care, which had a substantial effect on hidden costs, particularly lost income.52 High levels of pain, fatigue and depression can commence early in PD progression resulting in long-term burdens for caregivers. This impact was emphasised by a study that showed high levels of strain among PD caregivers and a significant association between this strain and quality of life as measured using the Parkinson's Disease Questionnaire (PDQ-39) $(p<0.001){ }^{53}$ This high impact was further demonstrated in a study of 1,211 PD caregivers who were compared with 6,055 control caregivers of individuals with other conditions..$^{54}$ Over 1 year, PD caregivers had significantly higher insurance, medical, prescription and out-of-pocket costs than other caregivers ( $p<0.01$ for all comparisons). In addition, PD caregivers had a significantly higher cumulative income loss over 5 years than other caregivers $(\$ 5,967$ versus $\$ 2,634, p=0.03)$.

The overall economic burden of PD is immense. A study based on 2010 figures, estimated a national economic burden of over $\$ 14.4$ billion/year in the USA (based on $\$ 22,800$ per patient) and medical expenses of $\$ 14.0$ billion, which was $\$ 8.1$ billion higher than a population without PD. ${ }^{14}$ This figure was expected to increase substantially with the number of patients with PD forecast to double by 2040. A further study ( $n=195$ ) conducted in 2013, estimated the lifetime cost of PD to be $€ 56,253 .{ }^{55}$ The top three components of this were productivity losses (67.7\%), pharmacotherapy (11.4\%) and home care (8.7\%). In particular, cognitive dysfunction and dementia in PD increase costs markedly. This was demonstrated in a study $(n=61)$ in which in patients with PD were monitored over a 9-year period; the cost for those with dementia was 3.3-fold higher than for those without it. ${ }^{56}$ This increase was largely a result of institutional care costs.

The burdens of PD on patients, caregivers and treatment providers are therefore extensive and affect an increasing and ageing population. Patients diagnosed with PD, have to live with the condition and modern treatment strategies suggest a normal lifespan, and as such require considerable allocation of resources. Much research and development are needed to reduce the lifelong burden of PD.

\section{Unmet needs in Parkinson's disease}

Understanding of PD has advanced considerably over the past 10-15 years but many urgent unmet needs remain and these contribute towards the burden the condition imposes. A summary of key unmet needs in PD is given in Table 1. The most fundamental of these needs is a better understanding of PD pathophysiology, which would lead to holistic models of treatment with multiple modes of action rather than 


\section{Table 1: Summary of key unmet needs in Parkinson's disease}

\begin{tabular}{|c|c|}
\hline Unmet need & Potential benefits \\
\hline Improved understanding of PD pathophysiology & $\begin{array}{l}\text { Development of neuroprotective therapies - limited by insufficient understanding } \\
\text { of cellular processes and inadequate animal models. Creation of treatment models } \\
\text { with multiple modes of action rather than single-target strategies leading to improved } \\
\text { treatment efficacy }\end{array}$ \\
\hline Reliable set of composite biomarkers & $\begin{array}{l}\text { Genetic, biochemical and imaging biomarkers would assist early detection, aid } \\
\text { diagnosis, monitor progression and indicate response to treatment }\end{array}$ \\
\hline Better combination of motor and non-motor outcome measures & Improved understanding of disease status and necessary treatments \\
\hline $\begin{array}{l}\text { More effective and tolerable therapies for both motor and non-motor } \\
\text { symptoms including non-dopaminergic motor treatments e.g. adenosine } \\
\text { receptor antagonists }\end{array}$ & Improve non-motor efficacy and address underlying disease \\
\hline More effective therapies for specifically treating non-motor symptoms & $\begin{array}{l}\text { Treat non-motor symptoms e.g. depression, cognition, pain, fatigue and GI issues that } \\
\text { severely affect patient QoL. These are largely not addressed by current treatments and } \\
\text { can emerge earlier than motor symptoms }\end{array}$ \\
\hline Multidisciplinary approach & $\begin{array}{l}\text { Providing coordinated care from multiple different medical specialities that would } \\
\text { improve disease detection and diagnosis and achieve optimal and coordinated } \\
\text { disease management }\end{array}$ \\
\hline
\end{tabular}

Gl = gastrointestinal; $P D=$ Parkinson's disease; $Q \mathrm{QL}=$ quality of life.

Table 2 Existing and proposed biomarkers for diagnosis and progression in Parkinson's disease

\begin{tabular}{|c|c|}
\hline Biomarker class & Existing and proposed biomarkers \\
\hline \multicolumn{2}{|l|}{ Clinical } \\
\hline Prodromal non-motor symptoms & $\begin{array}{l}\text { RBD, late onset hyposmia/anosmia, episodic major depression, constipation, excessive daytime somnolence, } \\
\text { fatigue, abnormal colour vision/visual perception, erectile dysfunction, pain (often unilateral), cognitive } \\
\text { impairment, hyperechogenicity, apomorphine }\end{array}$ \\
\hline Motor symptoms & Tremor, postural instability, dyskinesias, micrographia \\
\hline Scales & UPDRS, Scopa Autonomic, PD Non-motor Symptoms Scale \\
\hline \multicolumn{2}{|l|}{ Genetic markers } \\
\hline Blood-based & GBA, LRRK2, $\alpha$-synuclein, Parkin, PINK1, DJ-1, hereditary forms (PARK1 to PARK11) \\
\hline \multicolumn{2}{|l|}{ Biochemical markers } \\
\hline Blood-based & $\begin{array}{l}\alpha \text {-synuclein, neuromelanin antibodies, oxidative and mitochondrial markers, uric acid and serum urate, EGF, } \\
\text { Apo A1 }\end{array}$ \\
\hline Cerebrospinal fluid-based & $\alpha$-synuclein, DJ-1, GBA activity, A $\beta-42$, neurofilaments, $\alpha$-synuclein, RT-QuIC \\
\hline Urine & 8-Hydroxydeoxyguanosine \\
\hline Faeces & Decreases in protective gut microbiota composition in PD (needs further validation) \\
\hline Imaging techniques & ${ }^{18}$ F-DOPA PET, ${ }^{123}$-beta-CIT SPECT, MIBG scintigraphy, functional imaging \\
\hline
\end{tabular}

None of the tests provide robust diagnostic proof in isolation. Multimodal validity is being tested. ${ }^{123}$-beta-CIT SPECT = IOdine (123) beta carboxymethoxy-3 beta-(4-iodophenyl) tropane single-photon emission computed tomography imaging; ${ }^{18} \mathrm{~F}-D O P A$ PET = ${ }^{18} \mathrm{~F}$-dihydroxyphenylalanine positron emission tomography; $A \beta-42=\mathrm{A}$ beta containing amyloid; Apo A1 = apolipoprotein A1; $\alpha$-synuclein RT-QuIC $=\alpha$-synuclein real time quaking-induced conversion assay; $D J-1=$ an oncogene associated with autosomal recessive early-onset Parkinson's disease; EGF = epidermal growth factor; GBA = glucocerebrosidase; $L R R K 2=$ leucine-rich repeat kinase 2; $M I B G={ }^{123}$-meta-iodobenzylguanidine; $P A R K=$ Parkinson's disease loci; $P D=$ Parkinson's disease; $P I N K 1=P T E N$-induced putative kinase 1; RBD = REM sleep behaviour disorder; REM = rapid eye movement; UPDRS = Unified Parkinson's Disease Rating Scales. Adapted from Titova et al. 2017, ${ }^{57}$ and Gerlach et al. 2008. ${ }^{58}$

single-target strategies. ${ }^{4}$ A blind 'tunnel vision' strategy of purely addressing the dopaminergic system is unlikely to provide any neuroprotective or generally effective treatment. It is for this reason that levodopa, a molecule that is effective in multiple neurotransmitter pathways remains the most effective drug for PD and a myriad of drugs tried and tested for neuroprotection have failed at a huge cost to the industry. ${ }^{37}$ Whilst characteristic changes in the brain, such as death of dopaminergic neurones in basal ganglia and Lewy bodies, are well known, the factors that initiate PD and mediate progression are largely unknown. The gut-brain hypothesis of PD aetiology is an interesting concept but considerable further evidence is needed before it can be accepted that the gut flora plays a key role in PD pathophysiology. ${ }^{30-33}$ Improved understanding could provide valuable insights for inhibiting or blocking these processes leading to improved treatments and outcomes.

A further unmet need is a larger and more accurate set of biomarkers to aid diagnosis, indicate PD stage, monitor progression and treatment response, predict trajectory and identify targets for treatment. ${ }^{57}$ Such biomarkers need to be multimodal to reflect the heterogeneity of PD. Biomarkers fall into several categories: clinical signs/tests, genetic, biochemical and imaging (Table 2). Clinical signs of motor and/or non-motor symptoms and the use of scales (e.g. Unified Parkinson's 
Disease Rating Scales [UPDRS]) remain the mostly widely used and reliable diagnostic and prognostic indicators. Genetic markers such as LRRK2; and mutations in glucocerebrosidase, PTEN-induced kinase 1 and the oncogene DJ-1 are only useful in limited numbers of cases or provide weak evidence. Biochemical markers such as levels of $\alpha$-synuclein and glucocerebrosidase are of variable use and some lack specificity for PD. ${ }^{41,43,57,58}$ Neuroimaging with PET scans have provided some evidence in patients suspected to have PD and are useful in the diagnostic sense but remain expensive and not widely available. ${ }^{59}$ Magnetic resonance imaging can demonstrate evidence of nigral, forebrain and subcortical white matter degeneration but remains of limited efficacy in PD diagnosis. Transcranial sonography can reveal increased nigral iron deposition in PD-susceptible healthy older individuals. In addition, single-photon emission tomography used with radioactive metabolic tracers to assess function at a receptor level can be used as surrogate markers for diagnosis, monitoring severity and progression. However, access to it is often limited and is too expensive for it to be adopted in clinically meaningful use in most parts of the world. None of the existing or currently proposed biomarkers are indicative or reliable when used in isolation, but collectively they can provide a strong indication that $\mathrm{PD}$ is present and possibly that progression has occurred. Much further development of existing and new PD biomarkers, which could be used in a collective multi-modal fashion is needed. ${ }^{57,58}$

Early detection of PD is an additional unmet need in PD. Until better genetic, biochemical and imaging biomarkers are developed and widely adopted, monitoring non-motor symptoms needs to become a critical part of detecting the PD before motor symptoms have appeared. ${ }^{58,60}$ Such early detection could enrich subject populations for neuroprotection trials when neuronal rescue or modification remains feasible. Furthermore, accurate evaluation of risk of developing motor PD from the 'prodromal' stage could also refine anticipatory care packages as well as ethical issues related to prodromal diagnosis of PD.

In terms of outcome measures to aid a value-based healthcare strategy, a combination of motor and outcome measures is required as a basic quality standard in PD. One study of consecutive patients with PD $(n=935)$ found a significant difference in severity classification between the Hoehn and Yahr (H\&Y) staging and the Non-motor Symptoms Burden (NMSB) scale (gamma=0.45; alpha's standard error $=0.032$ ) ${ }^{61}$ For more reliable severity grading in future, the authors proposed a combined approach in which NMSB levels are added to standard motor assessments as otherwise, assessments continue to focus on the motor syndrome of PD alone. A problem, therefore, is that such patients may have excellent motor response to the tested drug or intervention yet continue to progress with non-motor symptoms. ${ }^{62}$ Development of tools for individual non-motor symptoms assessment in PD also needs to keep pace with the motor research in PD. As an example, adequate assessment of pain is also an unmet need in PD. This could be addressed using scales such as the Kings Parkinson's Disease Pain Scale, a 14-item scale enabling various types of pain to be rated. A study of people with PD $(n=178)$ and matched controls $(n=83)$ showed that this scale is reliable and valid for rating various types of pain, and correlated well with other measures of pain and with scales of motor, non-motor and quality of life measures..$^{63} \mathrm{~A}$ recent review of grading scales in PD concluded that people with PD can be adequately assessed according to their needs using multiple different scales. ${ }^{64}$ They also believed that in the absence of good biomarkers for quantitative standardised information, a comprehensive method for grading the severity of PD manifestations is desirable. Assessing outcomes in PD therefore should be adopted in a value-based manner adding to the concept of value-based healthcare.
An outcomes 'scorecard' for PD is a feasible option where motor, non-motor and quality of life measures could be compositely presented allowing a full picture of the patient to be managed rather than the piecemeal motor-only approach that is currently in use..$^{65}$

The widespread use of digital technology is another unmet need in PD. This may aid the development of improved systems that enable more precise motor measurements and simultaneous monitoring of multiple patient parameters, although large scale validation is needed. ${ }^{66}$ In recent years there have been substantial advances in miniaturised and wearable technologies enabling the continuous capture of multiple motor, and aspects of non-motor, symptoms of PD. These measures have the potential to aid accurate diagnosis and more precisely monitor progression and treatment response..$^{67}$ To help achieve this, the International Parkinson and Movement Disorders Society Task Force on Technology is encouraging the establishment of open standard platforms for technology-based measurements and treatments by medical regulatory bodies such as the Food and Drug Administration and the European Medicines Agency. ${ }^{67}$

A further possible emerging technological benefit in PD comes from the analysis of 'big data' which is generated from various sources, such as data from well characterised cohort studies, clinical examinations, genetic and metabolic analyses including genotyping, imaging, the 'Internet of things' and wearable devices. ${ }^{67-69}$ For example, an analysis of extensive data collected in the Parkinson's Progression Markers Initiative (complex imaging, genetics, clinical and demographic data) used machine learning-based classification to predict PD. This analysis predicted PD in individuals with $>96 \%$ sensitivity and specificity and was $>80 \%$ accurate without the inclusion of UPDRS data. ${ }^{69}$ Big-data analysis can also utilise test data collected from patients' smartphones. ${ }^{70}$ It has the potential to aid PD screening, determine phenotype, provide insights into pathogenesis and identifying existing drugs that would benefit people with PD. ${ }^{71,72}$ An additional technology, virtual reality (VR), has been shown to improve balance and gait training in people with PD. A recent systematic review of 12 studies involving 419 participants found significant improvements in the Berg Balance Scale $(p<0.0001)$, Timed Up and Go Test $(p=0.04)$ and stride length $(p=0.0004)$ when comparing patients who received VR training versus controls. ${ }^{73}$

A major unmet need in PD results from most attention and the focus of treatments having been directed towards relief of the characteristic motor symptoms, rest tremor, rigidity, bradykinesia and postural instability. ${ }^{74}$ The current frequently-used dopamine replacement therapies are symptomatic and aim to increase dopaminergic activity in the striatum and comprise levodopa, dopamine agonists, monoamine oxidase B inhibitors and catechol-o-methyl transferase inhibitors, reuptake inhibitors as well as mixed transmitter active drugs (safinamide). ${ }^{75}$ Adverse events and falling clinical benefit limit the efficacy of these drugs over long periods, except for levodopa. However, levodopa, the gold standard, is frequently associated with end-of-dose 'wearing-off' leading to dyskinesias if used in high doses, often necessitating adjunctive or alternative therapy including varying modes of delivery of the drug (for example, transdermal, subcutaneous, or intrajejunal). Many non-dopaminergic motor, as well as non-motor, treatments in PD such as adenosine receptor antagonists, are in development ${ }^{7}$ but at present, remain an unmet need. This leaves a substantial need for more effective and well tolerated therapies for both motor and particularly non-motor symptoms, the latter remaining a key challenge to modern treatment of PD as articulated in the recently published Movement Disorder Society non-motor symptoms task force document. ${ }^{49}$ 
The development of neuroprotective therapies, a key unmet need in $\mathrm{PD}$, however, has been limited by a range of factors and has led to a succession of failures of molecules tested at a huge cost to industry over the years. The reasons for failure are multiple and include a lack of understanding of the multi-faceted and multi-neurotransmitter cellular processes involved in PD, and inadequate animal models, which do not reflect the progressive motor and non-motor natural history of PD, and crucially, starting trials at a motor stage of PD and thus ignoring the sometimes protracted and non-motor dominant prodromal stage of PD when molecular processes related to pathophysiology of PD have already started. ${ }^{10,50}$ Many highly publicised trials have thus failed, and most recently the GDNF (glial cell line-derived neurotrophic factor) trial, while showing great improvements in functional imaging with PET scans, showed no beneficial effect in patients even after 80 weeks follow-up. ${ }^{7,77}$

A reverse side of the coin is also the lack of high-quality evidence to manage PD in the most advanced and palliative stage, taking into account the multi-morbidity as well as frailty of ageing. A recent 'call to action' highlighted a further unmet need by stating that most of the clinical and research attention in PD is directed towards the mild to moderate stages but the advanced stages are neglected. ${ }^{74}$ In these later stages, usually in H\&Y stage $\mathrm{V}$, patients are often unable to attend clinics, are institutionalised and become lost to follow-up. Greater awareness and research into advanced and specifically palliative PD are needed to help this highly disabled and dependent patient population. Recent initiatives such as the palliative care task force of the Movement Disorders Society or the OPTCARE Neuro study are welcome positive actions in this regard. ${ }^{78}$

A further call from an international panel stressed that the complexity and heterogeneity of PD necessitates collaboration and data sharing across institutions and organisations. ${ }^{79}$ This would aid understanding of PD progression and help identify targets for effective disease-modifying therapies.

There are therefore, many unmet needs in PD in spite of major advances in motor treatment and knowledge regarding pathophysiology. A survey of patients with H\&Y stage I-III PD $(n=81)$ and caregivers of people with PD with H\&Y stage IV-V PD revealed several unmet needs. ${ }^{16}$ In particular, patients and caregivers wanted better treatments for motor symptoms and stated that the current treatments do not adequately alleviate non-motor symptoms. People with PD also felt that their concerns and anxieties were not taken seriously by physicians and there was insufficient two-way communication with them. A further patient survey $(n=500)$ found that patients thought general practitioners offered less information than they needed and wanted improved access to specialist care..$^{15}$ This theme was supported by more recent opinion that treatment of non-motor symptoms remains a key unmet need..$^{80}$ There is an evidence base for treating some non-motor symptoms such as pain, dementia, sleep dysfunction and constipation, but little information supports treatment of other symptoms such as anxiety, apathy, fatigue and insomnia. This evidence base has expanded substantially in recent years, but treatment options remain limited and some are impacted by adverse events. ${ }^{49}$

Treating non-motor symptoms is key to maintaining quality of life in both patients and carers. ${ }^{46}$ In addition, personalised medicine needs to be established for patients with PD. This includes the 'circle of personalised medicine' including lifestyle advice, body weight, exercise, complementary medicine, as well as endophenotypes of PD and would take account of individual patient parameters and genetic factors. ${ }^{60,81}$

Finally, various neurologists and patient groups have commented that an integrated multidisciplinary approach in both diagnosis and management is an unmet and crucial need in PD. ${ }^{82,83}$ This was highlighted by patient $(n=40)$ and caregiver $(n=20)$ opinions given in a study involving focusgroup discussions. ${ }^{84}$ Participants identified a lack of multidisciplinary collaboration to help in various aspects of their lives and this was considered to be a major bottleneck in the current healthcare system. such multidisciplinary operation in healthcare could provide patientcentred care. Multidisciplinary rehabilitation programmes in PD have shown some short-term benefits for patients, although high-level evidence supporting them is very limited..$^{85}$ Multidisciplinary care is achieved by the coordinated operation of various specialities, in particular PD specialist nurses who have various support roles in PD management including educating patients and families, medication use, clinical assessments, palliative care, perioperative care, and assessment and management of non-motor symptoms..$^{83}$ Greater awareness of PD and involvement of different medical disciplines have the potential to improve PD detection and ensure the disease is appropriately managed from an early stage.

\section{Conclusion}

There are many reasons for optimism in the understanding and management of PD in spite of the burden of unmet needs. Knowledge of PD pathophysiology is rapidly expanding and diagnostic methods are steadily improving. Whilst disease-modifying therapies are not yet a reality, some unmet needs can be addressed and 'slowing or altering' the progression pattern in PD may be a reality soon. Improved methods for the delivery of advanced therapies is already available and transforming the lives of many people with PD. More could be done to improve diagnostic protocols and recognise non-motor symptoms at an earlier stage to initiate suitable palliative medication and other treatments such as non-pharmacological interventions, including exercise as well as yoga and mind training. Such interventions could, in theory, delay progression and improve quality of life for both patients and their caregivers. Therefore, a more holistic approach that includes greater awareness and recognition of non-motor symptoms and readiness to treat them alongside continuing efforts to identify effective means of inhibiting the pathological process have the strong potential to improve present and future outcomes in PD. $\square$
1. GBD 2016 Parkinson's Disease Collaborators. Global, regional, and national burden of Parkinson's disease, 1990-2016: a systematic analysis for the Global Burden of Disease Study 2016. Lancet Neurol. 2018;17:939-53.

2. Athauda D, Foltynie T. Challenges in detecting disease modification in Parkinson's disease clinical trials. Parkinsonism Relat Disord. 2016:32:1-11.

3. Kalia LV, Kalia SK, Lang AE. Disease-modifying strategies for Karkinson's disease. Mov Disord. 2015:30:1442-50.

4. Chaudhuri RK, Bhidayasiri R, van Laar T. Unmet needs in Parkinson's disease: new horizons in a changing landscape. Parkinsonism Relat Disord. 2016;33(Suppl 1):S2-8.

5. Rocca WA. The burden of Parkinson's disease: a worldwide perspective. Lancet Neurol. 2018;17:928-9.

6. Rocca WA. The future burden of Parkinson's disease. Mov
Disord. 2018:33:8-9.

7. Whone A, Luz M, Boca M, et al. Randomized trial of intermittent intraputamenal glial cell line-derived neurotrophic factor in Parkinson's disease. Brain. 2019;142:512-25.

8. Oertel WH. Recent advances in treating Parkinson's disease. F1000Res. 2017;6:260.

. Sardi SP, Simuni T. New era in disease modification in Parkinson's disease: review of genetically targeted Parkinson's disease: review of genetically targeted
therapeutics. Parkinsonism Relat Disord. 2019:59:32-8.

therapeutics. Parkinsonism Relat Disord. 2019;59:32-8.
. Stocchi F. Therapy for Parkinson's disease: what is in the p. Stocchi F. Therapy for Parkinson's disease: Wh
pipeline? Neurotherapeutics. 2014;11:24-33.

1. Chaudhuri KR, Odin P, Antonini A, Martinez-Martin P. Parkinson's disease: the non-motor issues. Parkinsonism Relat Disord. 2011;17:717-23.

12. Chaudhuri KR, Antonini A, Robieson WZ, et al. Burden of non- motor symptoms in Parkinson's disease patients predicts improvement in quality of life during treatment with levodopa-carbidopa intestinal gel. Eur J Neurol. 2019;26:581-43.

13. Bovolenta TM, de Azevedo Silva SM, Arb Saba R, et al. Systematic review and critical analysis of cost studies Systematic review and critical analysis of cost studies
associated with Parkinson's disease. Parkinson's Disease. 2017;2017:3410946.

14. Kowal SL, Dall TM, Chakrabarti R, et al. The current and projected economic burden of Parkinson's disease in the United States. Mov Disord. 2013;28:311-8.

15. Buetow $S$, Giddings $L S$, Williams $L$, et al. Perceived unmet needs for health care among Parkinson's Society of New Zealand members with Parkinson's disease. Parkinsonism Relat Disord. 2008;14:495-500

16. Hatano T, Kubo SI, Shimo Y, et al. Unmet needs of patients 
with Parkinson's disease: interview survey of patients and caregivers. I Int Med Res. 2009:37:717-26.

17. Poewe W, Antonini A, Zillmans JC, et al. Levodopa in the treatment of Parkinson's disease: an old drug still going strong. Clin Interv Aging. 2010;5:229-38.

18. Port $B$. How the retreat of big pharma leads to lost opportunities for Parkinson's drug discovery, 2017. Available at: https://medium.com/parkinsons-uk/how-the-retreat-ofbig-pharma-leads-to-lost-opportunities-for-parkinsons-drug discovery-371b5e295be2 (accessed 29 April 2019)

19. Solis M. Back to basics: striving to stall Parkinson's disease progression, 2017. Available at: www.pharmaceutical-journal. com/news-and-analysis/features/back-to-basics-strivingto-stall-parkinsons-disease-progression/20203542.article (accessed 29 April 2019)

20. Dexter DT, Jenner P. Parkinson disease: from pathology to molecular disease mechanisms. Free Radic Biol Med. 2013;62:132-44.

21. Van Den Eeden SK, Tanner CM, Bernstein AL, et al. Incidence of Parkinson's disease: variation by age, gender, and race/ of Parkinson's disease: variation by age, gender
ethnicity. Am J Epidemiol. 2003:157:1015-22.

22. Gillies GE, Pienaar IS, Vohra S, Qamhawi Z. Sex differences in Parkinson's disease. Front Neuroendocrinol. 2014;35:370-84. 3. Savica R, Grossardt BR, Bower JH, et al. Time trends in the incidence of Parkinson Disease. JAMA Neurol. 2016;73:981-9.

24. Bellou V, Belbasis L, Tzoulaki I, et al. Environmental risk factors and Parkinson's disease: an umbrella review of meta-analyses Parkinsonism Relat Disord. 2016;23:1-9.

25. Mollenhauer B, Zimmermann J, Sixel-Doring F, et al. Baseline predictors for progression 4 years after Parkinson's disease diagnosis in the De Novo Parkinson Cohort (DeNoPa). Mov Disord. 2019;34:67-77.

26. Morozova N, O'Reilly EJ, Ascherio A. Variations in gender ratios support the connection between smoking and Parkinson's disease. Mov Disord. 2008:23:1414-9.

27. Pezzoli G, Cereda E. Exposure to pesticides or solvents and risk of Parkinson disease. Neurology. 2013;80:2035-41.

28. Vlaar T, Kab S, Schwaab Y, et al. Association of Parkinson's disease with industry sectors: a French nationwide incidence study. Eur J Epidemiol. 2018;33:1101-11.

29. Weisskopf MG, Weuve J, Nie H, et al. Association of cumulative lead exposure with Parkinson's disease. Environ Health Perspect. 2010;118:1609-13.

30. Miraglia F, Colla E. Microbiome, Parkinson's disease and molecular mimicry. Cells. 2019;8:222.

31. Mulak A, Bonaz B. Brain-gut-microbiota axis in Parkinson's disease. World I Gastroenterol. 2015;21:10609-20.

32. Rietdijk CD, Perez-Pardo P, Garssen J, et al. Exploring Braak's hypothesis of Parkinson's disease. Front Neurol. 2017;8:37.

33. Campos-Acuna J, Elgueta D, Pacheco R. T-cell-driven inflammation as a mediator of the gut-brain axis involved in inflammation as a mediator of the gut-brain axis in

34. Lionnet A, Leclair-Visonneau L, Neunlist M, et al. Does Parkinson's disease start in the gut? Acta Neuropathol. 2018;135:1-12.

35. Marder K, Levy G, Louis ED, et al. Familial aggregation of early- and late-onset Parkinson's disease. Ann Neurol. 2003;54:507-13

36. Zhang PL, Chen Y, Zhang $\mathrm{CH}$, et al. Genetics of Parkinson's disease and related disorders. J Med Genet. 2018;55:73-80.

37. Chaudhuri KR, Jenner P. Two hundred years since James Parkinson's essay on the shaking palsy. Have we made progress? Insights from the James Parkinson's 200 years course held in London, March 2017. Mov Disord. 2017;32:1311-5.

38. Alcalay RN, Levy OA, Waters CC, et al. Glucocerebrosidase activity in Parkinson's disease with and without GBA mutations Brain. 2015:138:2648-58.

39. Correia Guedes L, Ferreira JJ, Rosa MM, et al. Worldwide frequency of G2019S LRRK2 mutation in Parkinson's disease: a systematic review. Parkinsonism Relat Disord. 2010;16:237-42.

40. Friesen EL, De Snoo ML, Rajendran L, et al. Chaperone-based therapies for disease modification in Parkinson's disease.
Parkinson's Disease. 2017;2017:5015307.

41. Balestrino R, Schapira AHV. Glucocerebrosidase and Parkinson disease: molecular, clinical, and therapeutic implications. Neuroscientist. 2018:24:540-59.

42. O'Regan G, deSouza RM, Balestrino R, Schapira AH. Glucocerebrosidase mutations in Parkinson disease. Parkinsons Dis. 2017;7:411-22.

43. Schapira AH, Chiasserini D, Beccari T, Parnetti L. Glucocerebrosidase in Parkinson's disease: insights into pathogenesis and prospects for treatment. Mov Disord. 2016;31:830-5

44. Titova N, Padmakumar C, Lewis SJG, Chaudhuri KR. Parkinson's: a syndrome rather than a disease? I Neural Transm (Vienna). 2017;124:907-14.

45. Fredericks D, Norton JC, Atchison C, et al. Parkinson's disease and Parkinson's disease psychosis: a perspective on the challenges, treatments, and economic burden. Am J Manag Care. 2017;23:S83-92.

46. Titova N, Chaudhuri KR. Nonmotor Parkinson's and future directions. Int Rev Neurobiol. 2017;134:1493-505.

47. Poewe W. Non-motor symptoms in Parkinson's disease. Eur J Neurol. 2008;15(Suppl 1):14-20

48. Hely MA, Reid WG, Adena MA, et al. The Sydney multicenter study of Parkinson's disease: the inevitability of dementia at 20 years. Mov Disord. 2008;23:837-44.

49. Seppi K, Chaudhuri KR, Coelho M, et al. Update on treatments for nonmotor symptoms of Parkinson's disease-an evidencebased medicine review. Mov Disord. 2019;34:180-98.

50. Titova N, Chaudhuri KR. Non-motor Parkinson disease: new concepts and personalised management. Med J Aust. 2018;208:404-9

51. Hiseman JP, Fackrell R. Caregiver burden and the nonmotor symptoms of Parkinson's disease. Int Rev Neurobiol. 2017:133:479-97.

52. Whetten-Goldstein $\mathrm{K}$, Sloan F, Kulas E, et al. The burden of Parkinson's disease on society, family, and the individual. J Am Geriatr Soc. 1997:45:844-9.

53. Oguh $\mathrm{O}$, Kwasny M, Carter J, et al. Caregiver strain in Parkinson's disease: national Parkinson Foundation Quality Initiative study. Parkinsonism Relat Disord. 2013;19:975-9.

54. Martinez-Martin P, Macaulay D, Jalundhwala YJ, et al. The long-term direct and indirect economic burden among Parkinson's disease caregivers in the United States. Mov Disord. 2019;34:236-45.

55. Zhao YJ, Tan LC, Au WL, et al. Estimating the lifetime economic burden of Parkinson's disease in Singapore. Eur J Neurol. 2013;20:368-74.

56. Vossius C, Larsen JP, Janvin C, Aarsland D. The economic impac of cognitive impairment in Parkinson's disease. Mov Disord. 2011:26:1541-4.

57. Titova N, Qamar MA, Chaudhuri KR. Biomarkers of Parkinson's disease: an introduction. Int Rev Neurobiol. 2017:132:183-96.

58. Gerlach $M$, Hendrich $A$, Hueber $R$, et al. Early detection of Parkinson's disease: unmet needs. Neurodegener Dis. 2008;5:137-9.

59. Gaenslen A, Berg D. Early diagnosis of Parkinson's disease. Int Rev Neurobiol. 2010;90:81-92.

60. Titova N, Chaudhuri KR. Palliative care and nonmotor symptoms in Parkinson's disease and Parkinsonism. Int Rev Neurobiol. 2017;134:1239-55.

61. Chaudhuri KR, Rojo JM, Schapira AH, et al. A proposal for a comprehensive grading of Parkinson's disease severity combining motor and non-motor assessments: meeting an unmet need. PLoS One. 2013;8:e57221.

62. Schapira AHV, Chaudhuri KR, Jenner P. Non-motor features of Parkinson disease. Nat Rev Neurosci. 2017:18:435-50.

63. Chaudhuri KR, Rizos A, Trenkwalder C, et al. King's Parkinson's disease pain scale, the first scale for pain in PD: an international validation. Mov Disord. 2015:30:1623-31

64. Martinez-Martin P, Chaudhuri KR. Comprehensive grading of Parkinson's disease using motor and non-motor assessments: addressing a key unmet need. Expert Rev Neurother.
2018;18:41-50

65. Sauerbier A, Qamar MA, Rajah T, Chaudhuri KR. New concepts in the pathogenesis and presentation of Parkinson's disease. Clin Med (Lond). 2016:16:365-70.

66. Odin P, Chaudhuri KR, Volkmann J, et al. Viewpoint and practical recommendations from a movement disorder specialist panel on objective measurement in the clinical management of Parkinson's disease. NPJ Parkinsons Dis. 2018:4:14.

67. Espay AJ, Bonato P, Nahab FB, et al. Technology in Parkinson's disease: challenges and opportunities. Mov Disord. 2016;31:1272-82

68. Cohen S, Bataille LR, Martig AK. Enabling breakthroughs in Parkinson's disease with wearable technologies and big data analytics. Mhealth. 2016;2:20.

69. Dinov ID, Heavner B, Tang M, et al. Predictive big data analytics: a study of Parkinson's disease using large, complex heterogeneous, incongruent, multi-source and incomplete observations. PLoS One. 2016:11:e0157077.

70. Prince J, Arora S, de Vos M. Big data in Parkinson's disease: using smartphones to remotely detect longitudinal disease phenotypes. Physiol Meas. 2018:39:044005.

71. Olsen AL, Riise T, Scherzer CR. Discovering new benefits from old drugs with big data-promise for Parkinson disease. JAMA Neurol. 2018;75:917-20

72. Zhou L, Verstreken P. Reprogramming neurodegeneration in the big data era. Curr Opin Neurobiol. 2018;48:167-73.

73. Wang B, Shen M, Wang YX, et al. Effect of virtual reality on balance and gait ability in patients with Parkinson's disease: a systematic review and meta-analysis. Clin Rehabil. 2019;269215519843174.

74. Agarwal S, Fleisher JE. Reaching those most in need - a call to action for advanced Parkinson's disease. Eur Neurol Rev. 2016;11:20-1.

75. Zahoor I, Shafi A, Haq E. Pharmacological treatment of Parkinson's disease. In: Stoker TB, Greenland JC (eds.), Parkinson's Disease: Pathogenesis and Clinical Aspects. Brisbane (AU): Codon Publications; 2018 Dec 21. Chapter 7. Available at: www.ncbi.nlm.nih.gov/books/NBK536726/ (accessed 12 June 19).

76. Kalia LV, Brotchie JM, Fox SH. Novel nondopaminergic targets for motor features of Parkinson's disease: review of recent trials. Mov Disord. 2013;28:131-44.

77. Whone AL, Boca M, Luz M, et al. Extended treatment with glial cell line-derived neurotrophic factor in Parkinson's disease. J Parkinsons Dis. 2019;9:301-13.

78. van Vliet LM, Gao W, DiFrancesco D, et al. How integrated are neurology and palliative care services? Results of a multicentre mapping exercise. BMC Neurol. 2016;16:63.

79. Stephenson D, Hu MT, Romero K, et al. Precompetitive data sharing as a catalyst to address unmet needs in Parkinson's disease. J Parkinsons Dis. 2015:5:581-94.

80. Taddei RN, Spinnato F, Jenner P. New symptomatic treatments for the management of motor and nonmotor symptoms of Parkinson's disease. Int Rev Neurobiol. 2017;132:407-52.

81. Titova N, Chaudhuri KR. Personalized medicine in Parkinson's disease: time to be precise. Mov Disord. 2017;32:1147-54

82. Goldman JG, Vernaleo BA, Camicioli R, et al. Cognitive impairment in Parkinson's disease: a report from a multidisciplinary symposium on unmet needs and future directions to maintain cognitive health. NPJ Parkinsons Dis. 2018;4:19.

83. Parashos GA. Multidisciplinary treatment of Parkinson's disease: current state and future directions. Clin Pract. 2012;9:189-98

84. van der Eijk M, Faber MJ, Al Shamma S, et al. Moving towards patient-centered healthcare for patients with Parkinson's disease Parkinsonism Relat Disord. 2011:17:360-4.

85. Johnston M, Chu E. Does attendance at a multidisciplinary outpatient rehabilitation program for people with Parkinson's disease produce quantitative short term or long term improvements? A systematic review. NeuroRehabilitation 2010;26:375-83 